\title{
Sensitivity Improvement of Acoustic Partial Discharge Detection Measurements through Wavelet Analysis
}

\author{
Demetres Evagorou, Patrick Janus, Mohamad Ghaffarian Niasar and Hans Edin \\ KTH Royal Institute of Technology \\ School of Electrical Engineering \\ Stockholm, Sweden
}

\begin{abstract}
Condition monitoring of power equipment is a vital step in extending the lifetime of existing equipment and reducing costs for utilities while minimizing the risk of unscheduled outages. Partial discharge (PD) monitoring has evolved as a reliable mean of determining deterioration in insulation systems. Acoustic emission detection techniques are usually utilized for PD detection mainly in oil-filled transformers offering the advantage of being immune to electrical noise and a method to localize PDs. In this work it was attempted to improve the sensitivity of acoustic measurements through wavelet analysis and estimation of the threshold value from actual measurements of the noise, which proved to be more effective compared to other estimation values. The analysis was performed on laboratory measurements from a $36 \mathrm{kV}$ condenser bushing known to exhibit PD activity acquired with a low cost PD acoustic sensor developed at KTH. As a next step the results have to be verified by online measurements, which can result in the addition of an onboard signal-processing box for improved sensitivity.
\end{abstract}

\section{Introduction}

With on-line PD measurements noisy measurements seem to be inevitable. Depending on the equipment under test and the type of sensor used different types of noise can be present in the field. In the case of transformers an acoustic sensor is commonly used as a way to locate the PD [1]. Although the employment of such a sensor produces measurements that are immune to electrical noises present in the field there still exists noises such as interferences that affect the signal. In this work a procedure that improves the sensitivity of acoustic measurements is examined based on the wavelet packets transform and estimation of the noise from measurements. In an effort to evaluate the improvement in sensitivity a simple experiment was designed in the high voltage laboratory of the Royal Institute of Technology (KTH) in Stockholm, Sweden. The data acquired using this setup was used to evaluate the performance of the proposed algorithm. The whole experimental setup that involved a $36 \mathrm{kV}$ GOE condenser bushing known to exhibit PD activity.

\section{Experimental setup}

In order to acquire real PD signals under laboratorycontrolled conditions a simple experiment was designed. A $36 \mathrm{kV}$ GOE condenser bushing, produced by $\mathrm{ABB}$ and previously used at Forskmark Nuclear Power plant, was used as the source of real PDs since it was known to exhibit PD activity. This bushing was fed from a high voltage source and electrical as well as acoustic PD measurements were synchronously acquired. In order to be able to calibrate and quantify the apparent charge in the PD pulses a commercially available instrument, the ICM-system (Insulation Condition Monitoring) from Power Diagnostix Systems $\mathrm{GmbH}[2]$ was employed. This system works according

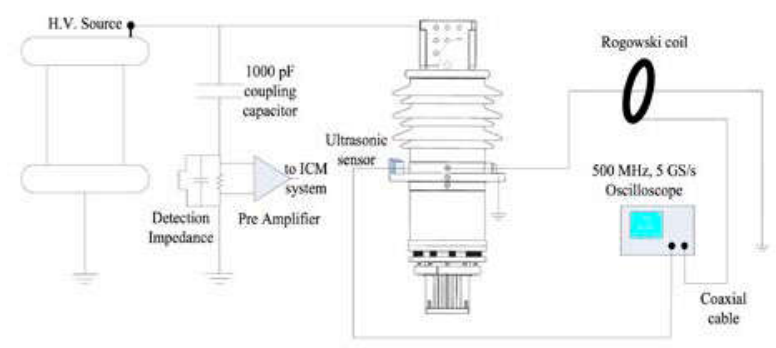

Figure 1. The experimental setup used to acquire the noise reference and PD activity signals of a $36 \mathrm{kV}$ condenser bushing. The bushing drawing was taken from the ABB website.

to the IEC-60270 standard [3] and was connected to a measurement impedance in series with a $1000 \mathrm{pF}$ coupling capacitor, followed by a preamplifier. A second set of measurements was taken with an acoustic sensor developed at KTH [4] and placed on the flange of the bushing. This sensor consists mainly of a contact acoustic sensor and a narrowband amplifier with a peak around $40 \mathrm{kHz}$. Both units were connected on the two channels of a PicoScope 3206B USB Oscilloscope, installed on a PC for data acquisition and storage. The data was acquired at an oversampled frequency of 62.5 $\mathrm{MHz}$ and in synchronization with the AC cycle.

\section{Wavelet Analysis}

The sensitivity improvement was based on the Wavelet Packets Transform (WPT) and its ability to compactly and adaptively represent the characteristic of the signal [5]. This procedure is a three-step procedure where the signal is transformed into a series of wavelet coefficients, a thresholding function acts on those 
coefficients, and finally the coefficients are projected back into the time domain. In this section each of these steps is briefly reviewed.

\subsection{Wavelet Packet Transform}

The ability of the wavelet packet transform (WPT) to represent the signal simultaneously in the time and frequency domain, subject to the Heisenberg's uncertainty principle renders it useful for analyzing transient, aperiodic and noisy signals [5]. The WPT is an extension of the Wavelet Transform (WT) that takes into account the characteristics of the signal under analysis. Starting from the WT the theory behind the WPT can be better explained.

The WT is based on multiresolution analysis, where orthogonal bases can be constructed using a scaling, $\varphi(\mathrm{t})$ and a wavelet function $\psi(\mathrm{t})$ [6]. The scaling and wavelet functions at scale $j$ and location $n$ are respectively given by

$$
\begin{aligned}
\phi_{j, n}(t) & =\frac{1}{\sqrt{2^{j}}} \phi\left(\frac{t-2^{j} n}{2^{j}}\right) \\
\psi_{j, n}(t) & =\frac{1}{\sqrt{2^{j}}} \psi\left(\frac{t-2^{j} n}{2^{j}}\right)
\end{aligned}
$$

The wavelet coefficients are the inner product of a signal $f$ with these scaling and wavelet functions given by

$$
\begin{aligned}
& a_{j}[n]=\left\langle f, \phi_{j, n}\right\rangle \\
& d_{j}[n]=\left\langle f, \psi_{j, n}\right\rangle
\end{aligned}
$$

The coefficients can be computed iteratively using a pair of conjugate mirror filters $h[n]$ and $g[n]$

$$
\begin{aligned}
& a_{j+1}[p]=\sum_{n=-\infty}^{\infty} h[n-2 p] a_{j}[n] \\
& d_{j+1}[p]=\sum_{n=-\infty}^{\infty} g[n-2 p] d_{j}[n]
\end{aligned}
$$

given by

$$
\begin{aligned}
& h[n]=\frac{1}{\sqrt{2}}\left\langle\phi\left(\frac{t}{2}\right), \phi(t-n)\right\rangle \\
& g[n]=\frac{1}{\sqrt{2}}\left\langle\psi\left(\frac{t}{2}\right), \phi(t-n)\right\rangle
\end{aligned}
$$

and

$$
g[n]=(-1)^{1-n} h[1-n] .
$$

where the approximation coefficients at level $a_{j}$ are further decomposed into approximation $a_{j+1}$ and detail coefficients $d_{j+1}$ at level $j+1$ up to a maximum decomposition level $J$. The filters $h[n]$ and $g[n]$ have low and high pass characteristics respectively and these coefficients represent different frequency content of the original signal but are full band signals themselves.

The scaling and wavelet functions in the WT are a priori chosen irrespective of the characteristics of the signal under analysis. This limitation is overcome through the use of the Wavelet Packet Transform, where the scaling and wavelet functions are chosen from a predetermined dictionary set according to an optimality criterion for the signal under examination. This is achieved by decomposing also the detail coefficients at scale $L+1$, position $p d_{L+1}^{p}$ into a set of approximation $d_{L+2}^{2 p}$ and detail coefficients $d_{L+2}^{2 p+1}$ at scale $L+2$. This adaptability of the WPT according to the signals characteristics results into a more compact representation, which can achieve better sensitivity improvement results through thresholding of the wavelet coefficients.

\subsection{Thresholding of wavelet coefficients}

The thresholding function applied on the wavelet coefficients plays a significant role on the performance of the whole algorithm. A number of schemes were proposed depending on the application at hand but two main functions exist, namely the soft and hard thresholding rules. In the hard thresholding method the coefficients below the threshold value, $T$, are set to zero while in the soft thresholding rule the coefficients above $T$ are also shifted towards zero by an amount $T$. As a general guidance the soft threshold gives smoother signal approximations while the hard threshold yields higher SNRs for the same $T$ values.

There exist a number of estimation procedures resulting in different values but the main issue is to find a simple and automated way to choose the threshold [7]. Such a theoretical attempt was performed by the universal threshold, given by $T=\sigma \sqrt{2 \log _{e} N}$, where $N$ is the length of the signal and $\sigma$ is calculated by median filtering of the coefficients at the finest resolution [8]. Usually this threshold tends to produce higher estimation risks and a multiscale threshold $T_{j}$ given by $T_{j}=\sigma \sqrt{2 \log _{e} N_{j}}$ ) where $N_{j}$ is the number of coefficients at scale $j$, can be used to lower the risk. This threshold takes into account the fact that the ratio of the higher value to the total number of wavelet coefficients decreases with increasing scale due to the fact that the total number of coefficients decreases.

In an attempt to improve the estimation a different approach was taken where the threshold value was estimated from on-line noise measurements [9]. In this work that procedure was expanded to employ the WPT in comparison with the WT originally used. Moreover the original field of application was electrically coupled measurements on power cables while acoustic measurements with application in transformers are dealt with here. The whole sensitivity improvement procedure is explained in the next section.

\section{Sensitivity improvement}


The whole sensitivity improvement procedure is outlined here as a four step procedure:

1. A noise reference reading is needed together with a PD signal. In the case of laboratory controlled conditions, such as the experimental setup in section 2 , the voltage is raised to a value well below the PD inception and several recordings of the noise are acquired. Subsequently the voltage is raised well above the inception voltage and PD activity recordings are taken. However in on-line measurements this procedure has to be modified and a circumvention can be the utilization of simultaneous measurements of two or more identical sensors in varying locations. One sensor is moved around until a position is found where the PD activity is highly attenuated and its reading is used as the noise reference. On the other hand the other sensor is placed at a position where the PD reading is maximized.

2. The noise reference signal is expanded into a series of wavelet coefficients through the use of the wavelet packets transform. The wavelet used is the Symmlets 8 and this choice is based on previous results and experiences [10]. The wavelet packet tree uses 10 levels of decomposition giving a total of 2047 nodes in the full decomposition tree. For each of these nodes the maximum value of the wavelet coefficients is stored and used as the threshold value for each node giving a node dependent thresholding estimator.

3. The PD signal is decomposed into a set of coefficients and Shannon's entropy is used as the cost function for best basis selection. The coefficients in the terminal nodes in this best basis are thresholded based on the estimation of the noise level in step 2 in that particular node.

4. The thresholded coefficients of the signal are transformed back into the time domain.

In the next section results from laboratory measurements using a calibration pulse as well as a condenser bushing with a nominal voltage of $36 \mathrm{kV}$ known to exhibit PD activity are used to evaluate this sensitivity improvement procedure.

\section{Results}

In order to test the sensitivity improvement procedure a calibration pulse of known magnitude $(50 \mathrm{pC})$ was injected into the measurement system and detected using the ICM system. The choice of a calibration pulse was made due to the fact that it appears in synchronization with the AC cycle (one pulse per cycle) and serves as a reference pulse. The acoustic sensor does not pick it up since it is a purely electrical signal but can be used to evaluate the proposed method prior to applying it on a real bushing.

Table 1 Sensitivity improvement for $50 \mathrm{pC}$ calibration pulse using wavelet and wavelet packets as well as different threshold estimation values. The $50 \mathrm{pC}$ calibration pulse corresponds to $195.4 \mathrm{mV}$ on the ICM.

\begin{tabular}{|c|c|c|c|c|}
\hline \multirow{2}{*}{$\begin{array}{c}\text { Wavelet Analysis } \\
\text { Type }\end{array}$} & \multicolumn{2}{|c|}{ Threshold } & \multirow{2}{*}{$\begin{array}{l}\text { SNR } \\
\text { (dB) }\end{array}$} & \multirow{2}{*}{$\begin{array}{l}\text { Noise } \\
\text { floor } \\
(\mathrm{mV})\end{array}$} \\
\hline & Function & Type & & \\
\hline \multirow{6}{*}{ Wavelet transform } & \multirow{3}{*}{ soft } & universal & 191 & 40 \\
\hline & & multiscale & 389 & 5 \\
\hline & & $\begin{array}{l}\text { noise } \\
\text { reference }\end{array}$ & 745 & 5 \\
\hline & \multirow{3}{*}{ hard } & universal & 182 & 40 \\
\hline & & multiscale & 390 & 10 \\
\hline & & $\begin{array}{l}\text { noise } \\
\text { reference }\end{array}$ & 748 & 8 \\
\hline \multirow{6}{*}{$\begin{array}{l}\text { Wavelet packets } \\
\text { transform }\end{array}$} & \multirow{3}{*}{ soft } & universal & 743 & 20 \\
\hline & & multiscale & 746 & 7 \\
\hline & & $\begin{array}{l}\text { noise } \\
\text { reference }\end{array}$ & 778 & 3 \\
\hline & \multirow{3}{*}{ hard } & universal & 745 & 10 \\
\hline & & multiscale & 766 & 7 \\
\hline & & $\begin{array}{l}\text { noise } \\
\text { reference }\end{array}$ & 769 & 10 \\
\hline Calibration pulse & ----------- & ------------- & 42 & 50 \\
\hline
\end{tabular}

The results of applying the wavelet and the wavelet packets transform on the calibration pulse are summarized in Table 1. Several different threshold estimation values are compared (explained in section 3.2) namely the universal, the multiscale and the estimated from noise reference measurements. The criteria used were the SNR and the noise level where the latter was defined as the level below which no pulse is detectable and the former given by

$$
\operatorname{SNR}_{d B}=10 * \log _{10}\left(\frac{\frac{1}{N} \sum_{n=0}^{N-1}(f[n]-\mu)^{2}}{\sigma^{2}}\right)
$$

Clearly the sensitivity improvement using the wavelet packet transform outperformed the wavelet transform, as expected, due to the adaptability of the former on the signal's characteristics. The estimation of the threshold value from noise reference gave the best results as far as SNR and noise floor level was concerned.

Next the sensitivity improvement procedure was applied on data collected from the $36 \mathrm{kV}$ condenser bushing at an applied voltage of $21.5 \mathrm{kV}$ and ambient conditions of $22^{\circ} \mathrm{C}$ with humidity of $52.1 \%$. The measurements were recorded for the ICM, the acoustic sensor and a High Frequency Current Transformer (HFCT) connected around the earthing of the equipment. The noise reference was collected at an applied voltage of $1.76 \mathrm{kV}$ well below the inception value of PDs and the results are shown in Table 2.

Contrary to what was expected the soft thresholding rule gives slightly better SNR results, probably due to its lower noise floor level. The choice of the threshold type for this application was based on the finding that the 
Table 2. Sensitivity improvement of the ICM and acoustic sensor measurements of PD activity from the bushing at an applied voltage of $21.5 \mathrm{kV}$.

\begin{tabular}{|l|l|c|c|}
\hline \multicolumn{1}{|c|}{ Signal } & $\begin{array}{c}\text { Threshold } \\
\text { function }\end{array}$ & $\begin{array}{c}\text { SNR } \\
\text { (dB) }\end{array}$ & $\begin{array}{c}\text { Noise } \\
\text { floor (mV) }\end{array}$ \\
\hline \multirow{3}{*}{ ICM } & soft & 816 & 20 \\
\cline { 2 - 4 } & hard & 794 & 10 \\
\cline { 2 - 4 } & original & 769 & 50 \\
\hline \multirow{3}{*}{ Acoustic sensor } & soft & 170 & 25 \\
\cline { 2 - 4 } & hard & 137 & 75 \\
\cline { 2 - 4 } & original & 118 & 300 \\
\hline
\end{tabular}

soft rule gave lower noise floor while the hard thresholding better preserved the amplitude of the pulses. Due to this property of amplitude preservation, the hard thresholding rule has been employed in accordance with [9], [10].

The result of the whole sensitivity improvement is shown in Figure 2 where the original acoustic signal is compared with the denoised signal using the noise measurement to estimate the threshold, as well as a reading from the HFCT, which is used as a reference. The results seem to be within acceptable limits as the two peaks recorded from the HFCT clearly appear in the denoised signal. Estimating the threshold value with the multiscale or universal threshold methods completely fails to denoise the signal, which highlights the improvement of the threshold estimation employed in this work.
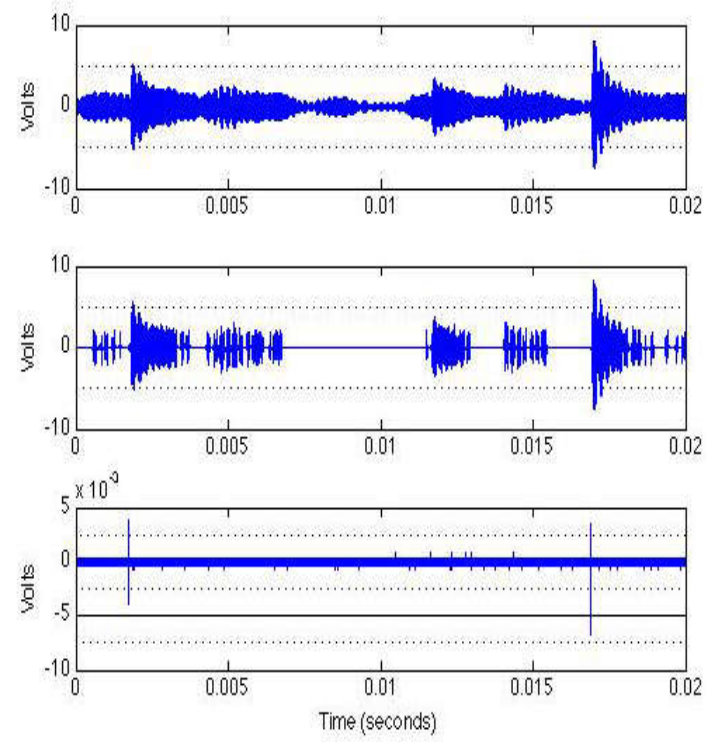

Figure 2. From top to bottom: (a) the PD signal from the condenser bushing at an applied voltage of $21.5 \mathrm{kV}$ using a contact acoustic sensor, (b) the denoised signal using hard thresholding with the value being estimated from a noise reference signal recorded at $1.76 \mathrm{kV}$ and (c) the same PD recorded using an HFCT sensor and used as a reference.

\section{Conclusions}

A sensitivity improvement procedure was presented here for PD detection using acoustic measurements. The strength of the procedure lies in the estimation of the noise from actual measurements and the employment of the wavelet packet transform. The procedure was tested under laboratory conditions with promising results and as a next step its performance in field conditions should be assessed.

\section{References}

[1] S. Markalous, S. Tenbolhen, and K. Feser, "Detection and Location of Partial Discharges in Power Transformers using acoustic and electromagnetic signals," IEEE Transactions on Dielectrics and Electrical Insulation, vol. 15, no. 6, pp. 1576-1583, 2008.

[2] "Power Diagnostix Systems GmbH." [Online]. Available: http://www.pdix.com/.

[3] IEC, IEC 60270 High-voltage test techniques Partial discharge measurements. 2000.

[4] P. Janus, "Acoustic Emission Properties of Partial Discharges in the time-domain and their applications," KTH Royal Institute of Technology, 2012.

[5] P. Addison, The Illustrated Wavelet Transform Handbook, 1st ed. Institute of Physics, 2002.

S. G. Mallat, "A theory for multiresolution signal decomposition: the wavelet representation," IEEE Transactions on Pattern Analysis and Machine Intelligence, vol. 11, no. 7, pp. 674-693, Jul. 1989.

X. Ma, C. Zhou, and I. J. Kemp, “Automated wavelet selection and thresholding for PD detection," IEEE Electrical Insulation Magazine, vol. 18, no. 2, pp. 37-45, Mar. 2002.

D. L. D. Donoho, I. M. Johnstone, and J. Johnstone, "Ideal Spatial Adaptation by Wavelet Shrinkage," Biometrika, vol. 81, no. 3, pp. 425-455, 1994.

H. Zhang, T. R. Blackburn, B. T. Phung, and D. Sen, "A novel wavelet transform technique for on-line partial discharge measurements. 2. Onsite noise rejection application," IEEE Transactions on Dielectrics and Electrical Insulation, vol. 14, no. 1, pp. 15-22, Feb. 2007.

[10] D. Evagorou, A. Kyprianou, P. L. Lewin, S. Andreas, V. Efthymiou, and G. E. Georghiou, "Evaluation of partial discharge denoising using the wavelet packets transform as a preprocessing step for classification," IEEE CEIDP 2008, pp. 387-390, 2008. 